\section{Actualización del bundle de reanimación inicial y monitorización integral de la perfusión tisular en la sepsis severa}

\author{
CARLOS ROMERO P. ${ }^{1}$, GLENN HERNÁNDEZ P. ${ }^{2}$
}

\section{Initial resuscitation bundle and monitoring tissue perfusion in severe sepsis}

Sepsis is a global health problem. Despite recent advances in understanding its pathophysiology and clinical trials testing potential new therapies, mortality remains unacceptably high. In fact, sepsis is the leading cause of death in non-coronary intensive care units around the world. However, during the past decade, some studies have highlighted that early recognition of sepsis and an appropriate initial approach are fundamental determinants of prognosis. A systematic approach to the harmful triad of sepsis-related hypotension, tissue hypoperfusion and organ dysfunction, with low-cost, easy to implement, and effective interventions, can significantly improve the chances of survival. In this article, we will update the evidence supporting the initial resuscitation bundle for patients with severe sepsis, and discuss the physiological basis for perfusion monitoring during septic shock resuscitation.

(Rev Med Chile 2013; 141: 1173-1181).

Key words: Intensive care units; Reperfusion; Resuscitation; Sepsis; Shock, septic.

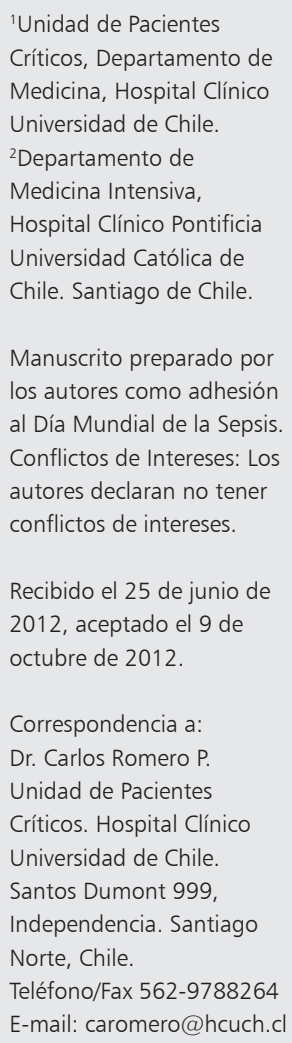

Recibido el 25 de junio de 2012, aceptado el 9 de octubre de 2012.

Correspondencia a: Dr. Carlos Romero P. Unidad de Pacientes Críticos. Hospital Clínico Universidad de Chile. Santos Dumont 999, Independencia. Santiago Norte, Chile.

Teléfono/Fax 562-9788264

E-mail: caromero@hcuch.cl

L a sepsis severa y el shock séptico son responsables de $20 \%$ de los ingresos a Unidades de Cuidados Intensivos (UCI) y constituyen la principal causa de muerte en UCI no coronarias ${ }^{1,2}$. En 2001, Angus y cols. ${ }^{1}$ estimaron que en Estados Unidos de Norteamérica podrían existir 750.000 casos anuales de sepsis severa, con una mortalidad asociada de 28,6\% y un impacto económico de 17 billones de dólares. En Chile, un estudio multicéntrico encontró una prevalencia de sepsis severa en pacientes críticos de $40 \%$, con una mortalidad asociada de $27 \%{ }^{3}$. Si hacemos una proyección, considerando que América Latina tiene una población aproximada de 580 millones de habitantes, el número de víctimas fatales anuales podría ser enorme. El enfrentamiento protocolizado de la nefasta triada asociada a la sepsis severa: hipotensión, hipoperfusión tisular y disfunción orgánica, puede generar una mejoría significativa en las posibilidades de supervivencia de los pacientes ${ }^{4-7}$.

Actualizaremos la evidencia que sustenta el bundle para la reanimación inicial de los pacien- tes con sepsis severa y expondremos los aspectos más relevantes sobre la evaluación de la perfusión tisular en la fase de reanimación avanzada del shock séptico.

\section{Bundle de reanimación inicial en la sepsis severa}

La estrategia del bundle consiste en incorporar un pequeño grupo de intervenciones basadas en evidencia, para mejorar diferentes procesos del cuidado de los pacientes críticos ${ }^{8}$. La Surviving Sepsis Campaign ha promovido un bundle para el reconocimiento precoz y la reanimación inicial de pacientes con sepsis severa-12 (Tabla 1). El bundle de reanimación tiene como objetivo estandarizar el cuidado inicial y asegurar que los enfermos reciban las intervenciones básicas que han demostrado mejorar los desenlaces, pero a la vez permitir un manejo individualizado de los pacientes ${ }^{13}$. Sus componentes se discuten a continuación: 
Tabla 1. Bundle de Reanimación Inicial: Este conjunto de medidas deben ser implementadas dentro de las primeras $6 \mathrm{~h}$ de diagnosticada una sepsis severa

1. Medir lactato plasmático o déficit de base si no dispone del primero

2. Tomar hemocultivos antes de iniciar la administración de antibióticos

3. Administración precoz y combinada de antibióticos de amplio espectro, dentro de $3 \mathrm{~h}$ de su llegada al Servicio de Emergencia o dentro de $1 \mathrm{~h}$ de su ingreso a una Unidad de Pacientes Críticos

4. En presencia de hipotensión y/o lactato $34 \mathrm{mmol} / \mathrm{L}$ o déficit de base $<-4 \mathrm{mmol} / \mathrm{L}$ :

a) Administre 2 litros de solución con cristaloides

b) Inicie noradrenalina cuando la hipotensión no responda a la reanimación inicial con volumen, con la finalidad de mantener una presión arterial media $\geq 65 \mathrm{mmHg}$

5. Si la hipotensión persiste a pesar de la resucitación con volumen y/o lactato $\geq 4 \mathrm{mmol} / \mathrm{L}$ o déficit de base $<-4 \mathrm{mmol} / \mathrm{L}$ :

c) Obtenga una medición de presión venosa central y asegure un valor $\geq 8 \mathrm{mmHg}$

d) Busque llevar la $\mathrm{SvcO}_{2}$ a un valor $\geq 70 \%$

*Adaptado de (02005 Surviving Sepsis Campaign and Institute for Healthcare Improvement.

\section{Medir lactato plasmático}

La hiperlactatemia puede ser causada por un aumento de producción aeróbica, anaeróbica o un aclaramiento retardado. En todo caso, siempre debe excluirse primero la hipoperfusión o la hipoxia. De cualquier manera e independientemente de la causa, las concentraciones de lactato tienen un valor pronóstico muy importante. Tanto el primer valor de lactato desde la urgencia, el lactato máximo, el clearance de lactato, o el tiempo en que el lactato se normaliza son predictivos de mortalidad $^{14}$.

El exceso o déficit de base es definido como la cantidad de ácido o base que se debería adicionar a la sangre para restaurar el $\mathrm{pH}$ a 7,40 asumiendo que la presión arterial de dióxido de carbono se mantiene en $40 \mathrm{mmHg}$. Este índice se altera únicamente cuando el trastorno ácido-base es de origen metabólico, así en la acidosis metabólica la producción excesiva de hidrogeniones va a ocasionar el consumo de bicarbonato generando un déficit de base (DB). Recientemente, un estudio francés documentó la utilidad del DB para identificar pacientes con hiperlactatemia. La presencia de un DB menor a $-4 \mathrm{mmol} / \mathrm{L}$ tuvo una buena sensibilidad $(91,1 \%)$ y especificidad $(88,6 \%)$ para identificar pacientes con valores de lactato $>3 \mathrm{mmol} / \mathrm{L}$ a su llegada a Urgencia ${ }^{15}$, situación que podría ser de ayuda en centros en que no se disponga de la determinación de lactato. Hernández y cols. ${ }^{16}$ observaron que aquellos pacientes con sepsis severa que presentaron hipotensión y requirieron la administración de drogas vasoactivas, pero que no desarrollaron hiperlactatemia (disfunción cir- culatoria persistente), tuvieron muy bajo riesgo de morir a 28 días, en comparación con aquellos pacientes que desarrollaron hipotensión e hiperlactatemia $>2,5 \mathrm{mmol} / \mathrm{L}$ (shock séptico clásico). Por otra parte, Nguyen y cols. ${ }^{17}$, en un análisis post hoc, observaron que los pacientes con un clearance de lactato $\geq 10 \%$ en las primeras $6 \mathrm{~h}$ tuvieron mayores probabilidades de sobrevivir. Jones y cols. ${ }^{18}$, mediante un estudio de no inferioridad, compararon una reanimación precoz guiada por metas orientadas a normalizar la saturación de oxígeno venosa central $\left(\mathrm{SvcO}_{2}\right)$ versus alcanzar un clearance de lactato venoso $\geq 10 \%$. El estudio demostró que ambas intervenciones fueron equivalentes en términos de sobrevida hospitalaria. Recientemente, un estudio aleatorio multicéntrico ${ }^{19}$, desarrollado en pacientes críticos que ingresaron con hiperlactatemia $(>3 \mathrm{mmol} / \mathrm{L})$, encontró que una estrategia de reanimación inicial dirigida a reducir los niveles de lactato arterial $\geq 20 \%$ cada 2 horas durante las primeras 8 horas de estadía en la UCI, redujo las disfunciones orgánicas evaluadas a las 72 horas y la mortalidad hospitalaria ajustada por factores de riesgo.

\section{Tomar hemocultivos antes de iniciar la administración de antibióticos}

Esta intervención no debe retardar el inicio de la antibioticoterapia empírica. Sus resultados servirán para ajustar el esquema iniciado según la sensibilidad del patógeno aislado, así como permitir cambios del esquema antimicrobiano cuando corresponda ${ }^{20}$. Probablemente el mayor impacto de esta medida consiste en conocer la 
flora local de cada nosocomio, para que en el manejo de infecciones asociadas a la atención en salud, el esquema antibiótico a emplear sea no sólo precoz, sino también adecuado, maximizando su beneficio.

\section{Administración precoz de antibióticos de amplio-espectro}

Debe iniciarse dentro de las primeras 3 horas de ingreso al Servicio de Urgencia o dentro de 1 $\mathrm{h}$ de ingreso a la Unidad de Pacientes Críticos, cuando no viene desde Urgencia. Adicionalmente, se deben realizar a la brevedad todos los esfuerzos necesarios (clínica, laboratorio e imágenes) para identificar el foco de la infección y establecer rápidamente si el paciente requerirá cirugía o drenaje percutáneo para control del mismo ${ }^{20}$.

Kumar y cols. ${ }^{21}$, en un importante estudio retrospectivo, observaron que por cada hora de retraso en la administración del antibiótico apropiado en pacientes con shock séptico existía una reducción significativa en las probabilidades de sobrevivir. Gaieski y cols. ${ }^{22}$ encontraron que el principal determinante de mortalidad en pacientes con sepsis severa y shock séptico, en quienes la reanimación precoz guiada por objetivos se inició en el departamento de emergencia, fue precisamente el retardo en la administración de un adecuado esquema antimicrobiano. Un análisis desarrollado por Barochia y cols. ${ }^{23}$ sobre los ensayos clínicos que han evaluado el empleo del bundle de reanimación inicial, destaca la administración precoz y apropiada de antibióticos como uno de sus elementos más consistente. Recientemente, Kumar y cols. ${ }^{24,25}$ han reportado que la implementación de una estrategia que incorpore la administración precoz y combinada de antibióticos con diferente mecanismo de acción para el patógeno responsable de la infección, se asocia a una reducción significativa de la mortalidad en pacientes con shock séptico.

\section{En presencia de hipotensión y/o lactato $\geq 4 \mathrm{mmol} / \mathrm{L}$}

a) Administre 2 litros de solución con cristaloides.

b) Inicie drogas vasoactivas cuando la hipotensión no responda a la reanimación inicial con volumen, con la finalidad de mantener una presión arterial media $(\mathrm{PAM}) \geq 65 \mathrm{mmHg}$.

El elemento crucial en las primeras horas de una sepsis severa es la hipovolemia relativa y/o absoluta que presentan los pacientes. Por ello la reposición del volumen circulante efectivo constituye el pilar fundamental de esta fase, con la finalidad de modular la respuesta inflamatoria y restaurar la perfusión tisular antes de que ocurra un daño irreversible ${ }^{26}$. El aporte precoz de fluidos ha demostrado mejorar significativamente parámetros macro hemodinámicos, metabólicos y la micro circulación en pacientes con sepsis severa, independiente del tipo de fluido administrado ${ }^{27,28}$.

Algunos estudios han documentado buenos resultado clínicos en pacientes con shock séptico en quienes se ha empleado como meta hemodinámica una $\mathrm{PAM} \geq 65 \mathrm{mmHg}^{29-32}$. No obstante, algunos pacientes con hipertensión arterial crónica pueden requerir mayores niveles de PAM para asegurar una adecuada presión de perfusión a los diferentes órganos, lo que debe ser determinado en forma individualizada y evaluado a través del comportamiento de los diferentes marcadores de oxigenación tisular.

Durante décadas ha existido un acalorado debate en relación a cuál debería ser la droga vasoactiva de primera elección en pacientes con shock séptico. Los dos contrincantes históricos han sido dopamina y noradrenalina. Varios estudios han reportado resultados preocupantes en relación a dopamina, mostrándola como una droga con menor acción vasopresora, con mayor incidencia de arritmias por su acción beta adrenérgica y asociada con un peor desenlace en pacientes con shock séptico ${ }^{33-36}$. No obstante, la evidencia era fundamentalmente circunstancial, por lo que se requería de mejores estudios para alcanzar una conclusión definitiva. En este contexto, la conferencia de consenso de la Surviving Sepsis Campaign del año 2008 recomendó el uso indistinto de dopamina o noradrenalina como vasopresor de primera línea ${ }^{20}$.

En 2010, dos estudios prospectivos aleatorios que compararon dopamina con noradrenalina, $y$ que incluyeron 252 y 1.679 pacientes respectivamente, encontraron una reducción no significativa de la mortalidad a favor de los pacientes tratados con noradrenalina. Sin embargo, ambos estudios documentaron un riesgo significativamente mayor de desarrollar arritmias supra ventriculares en los pacientes que recibieron dopamina ${ }^{37,38}$. Recientemente, De Backer y cols. ${ }^{39}$, desarrollaron un metaanálisis de 11 estudios (un total de 2.768 pacientes) que compararon dopamina con noradrenalina en 
shock séptico. El análisis de los 6 ensayos de mejor calidad metodológica, prospectivos y aleatorios, concluyó que el riesgo de muerte es significativamente mayor con el uso de dopamina. En base a la información existente en la actualidad, noradrenalina debe ser considerada como el vasopresor de primera elección en pacientes con shock séptico ${ }^{40}$.

\section{Si la hipotensión persiste a pesar de la} resucitación con volumen $y / o$ lactato $\geq 4 \mathrm{mmol} / \mathrm{L}$

c) Obtenga una medición de presión venosa central (PVC) y asegure un valor $\geq 8 \mathrm{mmHg}$.

d) Busque llevar la $\mathrm{SvcO}_{2}$ a un valor $\geq 70 \%$.

Por todos es conocido que los índices estáticos basados en la determinación de la precarga ventricular (PVC o presión de oclusión en la arteria pulmonar $[\mathrm{POAP}]$ ) no pueden predecir la respuesta hemodinámica al aporte de fluidos. En la UCI, considerando que la mayoría de los pacientes se encuentran en ventilación mecánica, se prefiere el empleo de índices dinámicos (variabilidad del volumen sistólico, variabilidad de la presión de pulso o la prueba de elevación de las extremidades inferiores) para identificar aquellos pacientes cuyo IC y PAM son dependientes de precarga ${ }^{41}$. Sin embargo, la PVC es una medición que en general se encuentra fácilmente disponible, y por ello puede servir como un elemento de orientación inicial para descartar que un paciente se encuentre francamente hipovolémico en las primeras horas de la reanimación ${ }^{42,43}$. Adicionalmente, es importante considerar que aunque una $\mathrm{SvcO}_{2} / \mathrm{SvmO}_{2}$ baja puede identificar un estado de hipodébito ${ }^{4}$, no es un parámetro confiable para predecir la respuesta a fluidos ${ }^{45}$.

En contraparte, debe evitarse caer en la tentación de guiar o perpetuar la administración de fluidos solamente por un valor aislado de PVC o índice dinámico, sin tomar en cuenta el contexto general ${ }^{46}$. A diferencia de lo que ocurre en la fase precoz de la reanimación, donde el aporte de fluidos ha mostrado generar una respuesta predecible en la perfusión global, la administración tardía de fluidos ( $>48 \mathrm{~h}$ ) no mejora la microcirculación ${ }^{27}$, e inclusive algunos investigadores han observado que un balance hídrico positivo y un nivel de PVC $>12 \mathrm{mmHg}$, se asocia a mayor riesgo de muerte $^{47,48}$.

En su trabajo clásico, Rivers y cols. ${ }^{29}$ lograron demostrar que la adecuada corrección de la $\mathrm{SvcO}_{2}$ a un valor $\geq 70 \%$ durante las primeras 6 horas del diagnóstico de una sepsis severa o shock séptico, produjo una reducción significativa de la mortalidad hospitalaria. Sin embargo, es importante destacar que este protocolo, con sus diferentes intervenciones, no ha sido estudiado ni ha demostrado efectividad o seguridad fuera del marco de las primeras 6 horas de diagnóstico de una sepsis severa.

En la fase precoz de una sepsis severa la $\mathrm{SvcO}_{2} /$ $\mathrm{SvmO}_{2}$, nos brinda información valiosa sobre el balance entre la entrega y el consumo de oxígeno en el organismo. Aunque la $\mathrm{SvcO}_{2}$ y la $\mathrm{SvmO}_{2}$ no son exactamente equivalentes, lo clínicamente relevante es que ambas siguen una tendencia similar por lo que pueden ser utilizadas en forma intercambiable durante la fase de reanimación inicial ${ }^{49}$.

Los aportes más trascendentes del trabajo de Rivers y cols. ${ }^{29}$ radican, por una parte, en que los investigadores fueron capaces de demostrar por primera vez la enorme influencia de la precocidad de las intervenciones sobre el desenlace alejado en pacientes con sepsis severa y, por otra, en que generaron un cambio en el paradigma de la reanimación desde un enfoque preocupado por la macro hemodinamia (hidráulico) a un abordaje guiado por metas de perfusión tisular (metabólico). El protocolo incluyó varias intervenciones aplicadas secuencialmente para la normalización de la $\mathrm{SvcO}_{2}$ (fluidos, glóbulos rojos, inótropos e intubación y ventilación mecánica), sin que se haya podido establecer aún el aporte específico de cada una de ellas en forma independiente. Hernández y cols. ${ }^{50}$ realizaron un estudio multicéntrico prospectivo en 103 pacientes críticos para establecer el impacto de una maniobra de sedación e intubación sobre la $\mathrm{SvcO}_{2}$. Los autores encontraron que todos los pacientes, sépticos y no sépticos, incrementaron significativamente el valor de $\mathrm{SvcO}_{2}$, sin ninguna intervención adicional y sin evidencia de que esto realmente represente un mejoramiento en la disoxia tisular. En la actualidad se encuentran en marcha 3 estudios prospectivos con distribución aleatoria en Estados Unidos de Norteamérica, Europa y Australia que pretenden despejar el aporte independiente de cada una de las intervenciones del protocolo original de Rivers, y establecer la mejor estrategia de reanimación durante las "golden hours" de la sepsis severa.

$\mathrm{La} \mathrm{SvcO}_{2}$ tiene limitaciones y si bien en las primeras horas de iniciado el shock séptico parece 
ser un buen reflejo del balance entre el aporte y el consumo de oxígeno, esta situación puede variar ostensiblemente después de la reanimación inicial cuando los enfermos se encuentran en la UCI. Diferentes investigadores han documentado que en estas circunstancias la $\mathrm{SvcO}_{2}$ puede estar normal o elevada a pesar de evidentes trastornos de la perfusión tisular ${ }^{51-53}$, reflejando un cambio evolutivo del proceso en el tiempo donde lo que comienza a predominar son las alteraciones de la extracción relacionadas con perturbaciones de la microcirculación y/o disfunción mitocondrial ${ }^{54,55}$.

\section{Monitoreo multimodal de la perfusión tisular durante la reanimación avanzada del shock séptico}

En esta fase se pierde el comportamiento armónico que se observa durante la reanimación inicial en los diferentes marcadores de perfusión $\operatorname{tisular}^{56}$. Por ende, la interpretación del apropiado balance entre las necesidades de aporte y consumo de oxígeno a los tejidos se vuelve compleja, dinámica y desafiante.

Aunque desde hace mucho tiempo los signos clínicos de mala perfusión periférica (llene capilar lento, frialdad distal de extremidades, livideces) han sido reconocidos como marcadores tempranos de inadecuada oxigenación tisular, su evaluación subjetiva ha dificultado su difusión y aplicación práctica. Afortunadamente, en los últimos años ha resurgido el interés por este tipo de monitoreo clínico en pacientes con sepsis severa o shock séptico ${ }^{57}$. Lima y cols. ${ }^{58}$ desarrollaron un interesante estudio fisiológico en 50 pacientes críticos, con la finalidad de establecer si la evaluación subjetiva de la perfusión periférica pudiera identificar pacientes con mayor disfunción metabólica u orgánica. Los pacientes que presentaron alteración de la perfusión periférica tuvieron mayor incidencia de hiperlactatemia y más disfunciones orgánicas a las $48 \mathrm{~h}$. Recientemente, nuestro grupo demostró que la pronta recuperación de la perfusión periférica evaluada clínicamente, en forma simple y no invasiva al lado de la cama del paciente, es un buen predictor de reanimación exitosa en pacientes con shock séptico ${ }^{59}$.

Por otra parte, Lamia y cols. ${ }^{60}$ han propuesto que la diferencia veno-arterial de dióxido de carbono $\left(\mathrm{CO}_{2}\right)$ podría ser considerada como un indicador de la suficiencia del flujo sanguíneo venoso para remover el dióxido de carbono producido por los tejidos periféricos. Vallé y cols. ${ }^{61}$ realizaron un estudio fisiológico en 50 pacientes consecutivos con shock séptico quienes permanecieron con hiperlactatemia $>2 \mathrm{mmol} / \mathrm{L}$ a pesar de tener una $\mathrm{SvcO}_{2} \geq 70 \%$. Los autores pudieron documentar que aquellos enfermos que al ingreso al estudio presentaron una diferencia entre la presión de $\mathrm{CO}_{2}$ venoso central y arterial $\geq 6 \mathrm{mmHg}\left(\Delta \mathrm{CO}_{2}\right)$, tuvieron un IC significativamente más bajo, menor clearance de lactato arterial a las $12 \mathrm{~h}$ y evidencia de mayor disfunción de órganos a las 24 h. Este interesante elemento de monitoreo metabólico, de fácil obtención y ampliamente disponible, podría complementar la información requerida para optimizar y consolidar la reanimación.

El advenimiento de la tonometría gástrica y su aplicación clínica en pacientes críticos a comienzos de la década 1990-99, hizo posible la evaluación de la perfusión regional del territorio esplácnico. La isquemia esplácnica ha sido asociada con el desarrollo de disfunción orgánica múltiple y mayor mortalidad ${ }^{62,63}$. En la actualidad el parámetro más evaluado es el $\mathrm{CO}_{2 \text { gap }}$, que es la diferencia entre el $\mathrm{CO}_{2}$ de la mucosa gástrica y la presión arterial de $\mathrm{CO}_{2}\left(\mathrm{PaCO}_{2}\right)^{64}$. Un $\mathrm{CO}_{2 \text { gap }}>20 \mathrm{mmHg}$ es indicativo de hipoperfusión esplácnica ${ }^{65}$. Lamentablemente, algunas limitaciones técnicas en el perfeccionamiento de este sistema de monitoreo han impedido su empleo rutinario; no obstante, podría tener un rol en la evaluación de pacientes que han normalizado sus parámetros macro hemodinámicos y de perfusión sistémica pero que todavía pudieran tener hipoperfusión regional (shock oculto) $)^{66,67}$.

En los últimos años, la espectroscopia del infrarrojo cercano (NIRS) ha emergido como una herramienta útil en la evaluación de la saturación tisular de oxígeno $\left(\mathrm{S}_{\mathrm{O}_{2}}\right)^{68}$. Sin embargo, la gran superposición de valores de $\mathrm{S}_{t} \mathrm{O}_{2}$ entre sujetos sanos y pacientes con shock séptico, han limitado su utilidad para la toma de decisiones individuales. Varios investigadores ${ }^{69,70}$ han planteado la determinación simultánea de la $\mathrm{S}_{t} \mathrm{O}_{2}$ en la eminencia tenar de pacientes con shock séptico junto con una maniobra dinámica denominada test de oclusión vascular con la finalidad de evaluar la reactividad microvascular e identificar enfermos con alto riesgo de complicaciones y peor desenlace. Lima y cols. ${ }^{71}$ han alertado sobre que las condiciones locales de la perfusión periférica pueden influen- 
ciar significativamente las mediciones de $\mathrm{S}_{t} \mathrm{O}_{2}$ en la eminencia tenar. Recientemente, Colin y cols. ${ }^{72}$ han encontrado un mejor rendimiento con la determinación de la $\mathrm{S}_{t} \mathrm{O}_{2}$ en los músculos masetero y deltoides en las primeras $6 \mathrm{~h}$ de reanimación del shock séptico.

La posibilidad actual de realizar un monitoreo óptico de la microcirculación, a través de imágenes con el Orthogonal Polarization Spectral (OPS) o Sidestream Dark-Field (SDF), permiten una visualización directa de las severas perturbaciones de la microcirculación a nivel sublingual, y sin duda alguna, han mejorado nuestra comprensión sobre estos fenómenos ${ }^{73,74}$. Se ha observado que los pacientes que mejoran precozmente su patrón microcirculatorio tienen una mayor posibilidad de supervivencia ${ }^{75,76}$. No obstante, la respuesta de la microcirculación a manipulaciones hemodinámicas es compleja y depende mucho de su estado basal o del tipo de intervención ${ }^{32,77-80}$. Un estudio prospectivo aleatorio que comparó una infusión de nitroglicerina intravenosa contra placebo durante la reanimación inicial de pacientes con sepsis severa o shock séptico, no demostró mejoría de la microcirculación sublingual evaluada por SDF. Llamativamente, la mortalidad hospitalaria del grupo que recibió nitroglicerina intravenosa fue $34,3 \%$ comparada con $14,2 \%$ del grupo placebo $^{81}$.

\section{Consideraciones finales}

El componente fundamental de la disfunción circulatoria en las primeras horas de una sepsis severa es la hipovolemia. La implementación precoz del bundle de reanimación inicial puede garantizar la entrega de las intervenciones esenciales de esta fase, tiene sustento biológico y cuenta con evidencia que respalda su aplicación. Durante la reanimación avanzada del shock séptico el comportamiento de los diferentes marcadores de oxigenación tisular es impredecible. En este escenario, todos los marcadores individuales tienen grandes limitaciones para reflejar con precisión el verdadero estado de la perfusión tisular, cuando son usados en forma aislada, y pueden llevar a interpretaciones apresuradas. Un abordaje integrador mediante un monitoreo multimodal de la perfusión que incluya parámetros clínicos, macrohemodinámicos, metabólicos y microcirculatorios, podría superar estas limitaciones favoreciendo la identificación del mecanismo biológico que predomine en cada caso en particular, y consecuentemente, la aplicación de la mejor estrategia terapéutica $^{82}$. Es importante enfatizar que muchas de las intervenciones que han demostrado generar un impacto significativo en los desenlaces clínicos no requieren de grandes inversiones de dinero, ni de tecnología sofisticada, sino de conocimientos fisiopatológicos, una actitud proactiva y la optimización del trabajo en equipo ${ }^{83}$.

\section{Referencias}

1. Angus DC, Linde-Zwirble WT, Lidicker J, Clermont G, Carcillo J, Pinsky MR. Epidemiology of severe sepsis in the United States: analysis of incidence, outcome, and associated costs of care. Crit Care Med 2001; 29: 130310.

2. Martin GS, Mannino DM, Eaton S, Moss M. The Epidemiology of Sepsis in the United States from 1979 through 2000. N Engl J Med 2003; 348: 1546-54.

3. Dougnac A, Mercado M, Cornejo R, Cariaga M, Hernández G, Andresen $\mathrm{M}$, et al. Prevalencia de sepsis grave en las Unidades de Cuidado Intensivo. Primer estudio nacional multicéntrico. Rev Med Chile 2007; 135: 62030.

4. Annane D, Bellissante E, Cavaillon JM. Septic Shock. Lancet 2005; 365: 63-78.

5. Kortgen A, Niederprum P, Bauer M. Implementation of an evidence-based "standard operating procedure" and outcome in septic shock. Crit Care Med 2006; 34: 943 49.

6. Castro R, Regueira T, Aguirre ML, Lanos OP, Bruhn A, Bugedo G, et al. An evidence-based resuscitation algorithm applied from the emergency room to the ICU improves survival of severe septic shock. Minerva Anestesiol 2008; 74: 223-31.

7. Shapiro NI, Howell MD, Talmor D, Lahey D, Ngo L, Buras J, et al. Implementation and outcomes of the Multiple Urgent Sepsis Therapies (MUST) protocol. Crit Care Med 2006; 34: 1025-32.

8. Institute for Healthcare Improvement: Bundle Up for Safety. http://www.ihi.org/IHI/Topics/Improvement/ ImprovementMethods/ImprovementStories/BundleUpforSafety.htm (consultado el 15/05/2012).

9. Levy MM, Pronovost PJ, Dellinger RP, Townsend S, Resar RK, Clemmer TP, Ramsay G. Sepsis change bundles: Converting guidelines into meaningful change in behavior and clinical outcome. Crit Care Med 2004; 32 (Suppl.): S595-97.

10. Gao F, Melody T, Daniels DF, Giles S, Fox S. The impact 
of compliance with 6-hour and 24-hour sepsis bundles on hospital mortality in patients with severe sepsis: a prospective observational study. Crit Care 2005; 9: 76470.

11. Nguyen HB, Corbett SW, Steele R, Banta J, Clark RT, Hayes SR, et al. Implementation of a bundle of quality indicators for the early management of severe sepsis and septic shock is associated with decreased mortality. Crit Care Med 2007; 35: 1105-12.

12. Levy MM, Dellinger RP, Townsend SR, Linde-Zwirble WT, Marshall JC, Schorr C, et al. The Surviving Sepsis Campaign: results of an international guideline-based performance improvement program targeting severe sepsis. Intensive Care Med 2010; 36: 222-31.

13. Romero CM. Seguridad y Calidad en Medicina Intensiva. Med Intensiva 2009; 33: 346-52.

14. Hernández G, Díaz MA, Horta P. El lactato en el contexto de la sepsis y el shock séptico. En Castro J, Hernández G, Bruhn A, Romero CM (editores). Sepsis y Falla Multiorgánica $3^{\circ}$ edición. Editorial Mediterráneo Ltda. 2011. Santiago-Chile. Páginas 247-252.

15. Montassier E, Batard E, Segard J, Hardouin JB, Martinage A, Le Conte P, Potel G. Base excess is an accurate predictor of elevated lactate in ED septic patients. Am J Emerg Med 2012; 30: 184-7.

16. Hernández G, Castro R, Romero CM, de la Hoz C, Angulo D, Aranguiz I, et al. Persistent sepsis-induced hypotension without hyperlactatemia: Is it really septic shock? J Crit Care 2011; 26: 435e9-e14.

17. Nguyen HB, Rivers EP, Knoblich BP, Jacobsen G, Muzzin A, Ressler JA, Tomlanovich MC. Early lactate clearance is associated with improved outcome in severe sepsis and septic shock. Crit Care Med 2004; 32: 1637-42.

18. Jones AE, Shapiro NI, Trzeciak S, Arnold RC, Claremont HA, Kline JA. Lactate Clearance vs Central Venous Oxygen Saturation as Goals of Early Sepsis Therapy. A Randomized Clinical Trial. JAMA 2010; 303: 739-46.

19. Jansen TC, van Bommel J, Schoonderbeek FJ, Sleeswijk Visser SJ, van der Klooster JM, Lima AP, et al. Early Lactate-Guided Therapy in Intensive Care Unit Patients A Multicenter, Open-Label, Randomized Controlled Trial. Am J Respir Crit Care Med 2010; 182: 752-61.

20. Dellinger RP, Levy MM, Carlet JM, Bion J, Parker MM, Jaeschke R, et al. Surviving Sepsis Campaign: International guidelines for management of severe sepsis and septic shock: 2008. Crit Care Med 2008; 36: 296-327.

21. Kumar A, Roberts D, Wood KE, Light B, Parrillo JE, Sharma S, et al. Duration of hypotension before initiation of effective antimicrobial therapy is the critical determinant of survival in human septic shock. Crit Care Med 2006; 34: 1589-96.
22. Gaieski DF, Mikkelsen ME, Band RA, Pines JM, Massone R, Furia FF, et al. Impact of time to antibiotics on survival in patients with severe sepsis or septic shock in whom early goal-directed therapy was initiated in the emergency department. Crit Care Med 2010; 38: 1045 53.

23. Barochia AV, Cui X, Vitberg D, Suffredini AF, O’Grady NP, Banks SM, et al. Bundled care for septic shock: An analysis of clinical trials. Crit Care Med 2010; 38: 668-78.

24. Kumar A, Safdar N, Kethireddy S, Chateau D. A survival benefit of combination antibiotic therapy for serious infections associated with sepsis and septic shock is contingent only on the risk of death: A meta-analytic/ meta-regression study. Crit Care Med 2010; 38: 1651-64.

25. Kumar A, Zarychanski R, Light B, Parrillo J, Maki D, Simon D, et al. Early combination antibiotic therapy yields improved survival compared with monotherapy in septic shock: A propensity-matched analysis. Crit Care Med 2010; 38: 1773-85.

26. Rivers EP, Jaehne AK, Eichhorn-Wharry L, Brown S, Amponsah D. Fluid therapy in septic shock. Curr Opin Crit Care 2010; 16: 297-308.

27. Ospina-Tascon G, Neves AP, Occhipinti G, Donadello $\mathrm{K}$, Büchele G, Simion D, et al. Effects of fluids on microvascular perfusion in patients with severe sepsis. Intensive Care Med 2010; 36: 949-55.

28. Pottecher J, Deruddre S, Teboul JL, Georger JF, Laplace $\mathrm{C}$, Benhamou D, et al. Both passive leg raising and intravascular volume expansion improve sublingual microcirculatory perfusion in severe sepsis and septic shock patients. Intensive Care Med 2010; 36: 1867-74.

29. Rivers EP, Nguyen HB, Havstad S, Ressler J, Muzzin A, Knoblich B,et al. Early goal-directed therapy in the treatment of severe sepsis and septic shock. N Engl J Med 2001; 345: 1368-77.

30. Varpula M, Tallgren M, Saukkonen K, Voipio-Pulkki LM, Pettilä V. Hemodynamic variables related to outcome in septic shock. Intensive Care Med 2005; 31: 1066-71.

31. Dünser MW, Ruokonen E, Pettilä V, Ulmer H, Torgersen C, Schmittinger CA. Association of arterial blood pressure and vasopressor load with septic shock mortality: a post hoc analysis of a multicenter trial. Crit Care 2009; 13: R181.

32. Dubin A, Pozo MO, Casabella CA, Pálizas Jr3 F, Murias $\mathrm{G}$, Moseinco MC, et al. Increasing arterial blood pressure with norepinephrine does not improve microcirculatory blood flow: a prospective study. Crit Care 2009; 13: R92.

33. Martin C, Papazian C, Perrin G, Saux P, Gouin F. Norepinephrine or dopamine for the treatment of hyperdynamic septic shock? Chest 1993; 103: 1826-31. 
34. Levy B, Dusang B, Annane D, Gibot S, Bollaert PE. Cardiovascular response to dopamine and early prediction of outcome in septic shock: A prospective multiplecenter study. Crit Care Med 2005; 33: 2172-77.

35. Sakr Y, Reinhart K, Vincent JL, Sprung CL, Moreno R, Ranieri VM, et al. Does dopamine administration in shock influence outcome? Results of the sepsis occurrence in acutely ill patients (SOAP) study. Crit Care Med 2006; 34: 589-97.

36. Boulain T, Runge I, Bercault N, Benzekri-Lefevre D, Wolf M, Fleury C. Dopamine therapy in septic shock: Detrimental effect on survival? J Crit Care 2009; 24: 575-82.

37. Patel GP, Grahe JS, Sperry M, Singla S, Elpern E, Lateef O, Balk RA. Efficacy and safety of dopamine versus norepinephrine in the management of septic shock. Shock 2010; 33: 375-80.

38. De Backer D, Biston P, Devriendt J, Madl C, Chochrad D, Aldecoa C, et al. Comparison of Dopamine and Norepinephrine in the Treatment of Shock. N Engl J Med 2010; 362: 779-89.

39. De Backer D, Aldecoa C, Njimi H, Vincent JL. Dopamine versus norepinephrine in the treatment of septic shock: A meta-analysis. Crit Care Med 2012; 40: 725-30.

40. Annane D. Physicians no longer should consider dopamine for septic shock! Crit Care Med 2012; 40: 981.

41. Magder S. Fluid status and fluid responsiveness. Curr Opin Crit Care 2010; 16: 289-96.

42. Vincent JL, Weil MH. Fluid challenge revisited. Crit Care Med 2006; 34: 1333-7.

43. Perner A, Haase N, Wiis J, White JO, Delaney A. Central venous oxygen saturation for the diagnosis of low cardiac output in septic shock patients. Acta Anaesthesiol Scand 2010; 54: 98-102.

44. Teboul JL, Hamzaoui O, Monnet X. SvO2 to monitor resuscitation of septic patients: let's just understand the basic physiology. Crit Care 2011; 15: 1005.

45. Magner SA, Georgiadis G, Cheong T. Respiratory variations in right atrial pressure predict response to fluid challenge. J Crit Care 1992; 7: 76-85.

46. Magder S, Bafaqeeh F. The clinical role of central venous pressure measurements. J Intensive Care Med 2007; 22: 44-51.

47. Murphy CV, Schramm GE, Doherty JA, Reichley RM, Gajic O, Afessa B, et al. The importance of fluid management in acute lung injury secondary to septic shock. Chest 2009; 136: 102-9.

48. Boyd JH, Forbes J, Nakada T, Walley KR, Russell JA. Fluid resuscitation in septic shock: A positive fluid balance and elevated central venous pressure are associated with increased mortality. Crit Care Med 2011; 39: 25965.
49. Reinhart K, Kuhn HJ, Hartog C, Bredle DL. Continuous central venous and pulmonary artery oxygen saturation monitoring in the critically ill. Intensive Care Med 2004; 30: 1572-78.

50. Hernández G, Pena H, Cornejo R, Rovegno M, Retamal J, Navarro JL, et al. Impact of emergency intubation on central venous oxygen saturation in critically ill patients: a multicenter observational study. Crit Care 2009; 13: R63.

51. Bracht $H$, Hänggi $M$, Jeker B, Wegmüller N, Porta $F$, Tüller D, et al. Incidence of low central venous oxygen saturation during unplanned admissions in a multidisciplinary intensive care unit: an observational study. Crit Care 2007; 11: R2.

52. van Beest PA, Hofstra JJ, Schultz MJ, Boerma EC, Spronk $\mathrm{PE}$, Kuiper MA. The incidence of low venous oxygen saturation on admission to the intensive care unit: a multi-center observational study in The Netherlands. Crit Care 2008; 12: R33.

53. Textoris J, Fouche' L, Wiramus S, Antonini F, Tho S, Martin C, Leone M. High central venous oxygen saturation in the latter stages of septic shock is associated with increased mortality. Crit Care 2011; 15: R176.

54. Fink M. Cytopathic hypoxia in sepsis. Acta Anaesthesiol Scand Suppl 1997; 110: 87-95.

55. Ince C. The microcirculation is the motor of sepsis. Crit Care 2005; 9 (Suppl 4): S13-19.

56. Boerma EC, Kuiper MA, Kingma WP, Egbers PH, Gerritsen RT, Ince C. Disparity between skin perfusion and sublingual microcirculatory alterations in severe sepsis and septic shock: a prospective observational study. Intensive Care Med 2008; 34: 1294-8.

57. van Genderen ME, van Bommel J, Lima A. Monitoring peripheral perfusion in critically ill patients at the bedside. Curr Opin Crit Care 2012; 18: 273-9.

58. Lima A, Jansen TC, van Bommel J, Ince C, Bakker J. The prognostic value of the subjective assessment of peripheral perfusion in critically ill patients. Crit Care Med 2009; 37: 934-8.

59. Hernández G, Pedreros C, Veas E, Bruhn A, Romero C, Rovegno M, et al. Evolution of peripheral vs metabolic perfusion parameters during septic shock resuscitation. A clinical-physiologic study. J Crit Care 2012; 27: 2838 .

60. Lamia B, Monnet X, Teboul JL. Meaning of arteriovenous $\mathrm{PCO}_{2}$ difference in circulatory shock. Minerva Anestesiol 2006; 72: 597-604.

61. Vallée F, Vallet B, Mathe O, Parraguette J, Maria A, Silva $\mathrm{S}$, et al. Central venous-to-arterial carbon dioxide difference: an additional target for goal-directed therapy in septic shock? Intensive Care Med 2008; 34: 2218-25. 
62. Gutiérrez G, Palizas F, Doglio G, Wainsztein N, Gallesio A, Pacin J, et al. Gastric intramucosal $\mathrm{pH}$ as a therapeutic index of tissue oxygenation in critically ill patients. Lancet 1992; 339: 195-9.

63. Marik PE. Gastric intramucosal pH. A better predictor of multiorgan dysfunction syndrome and death than oxygen-derived variables in patients with sepsis. Chest 1993; 104: 225-9.

64. Gutiérrez G. A Mathematical Model of Tissue-Blood Carbon Dioxide Exchange during Hypoxia. Am J Respir Crit Care Med 2004; 169: 525-33.

65. Levy B, Gawalkiewicz P, Vallet B, Briancon S, Nace L, Bollaert PE. Gastric capnometry with air-automated tonometry predicts outcome in critically ill patients. Crit Care Med 2003; 31: 474-80.

66. Poeze M. Monitoring global volume-related hemodynamic or regional variables after initial resuscitation: What is a better predictor of outcome in critically ill septic patients? Crit Care Med 2005; 33: 2494-500.

67. Antonelli M, Levy M, Andrews PJ, Chastre J, Hudson LD, Manthous C, et al. Hemodynamic monitoring in shock and implications for management. International Consensus Conference, Paris, France, 27-28 April 2006. Intensive Care Med 2007; 33: 575-90.

68. Angulo M, López A, Hurtado J. Monitoreo de la oxigenación tisular. En Castro J, Hernández G, Bruhn A, Romero CM (editores). Sepsis y Falla Multiorgánica $3^{\circ}$ edición. Editorial Mediterráneo Ltda. 2011. SantiagoChile. Páginas 272-281.

69. Payen D, Luengo C, Heyer L, Resche-Rigon M, Kerever S, Damoisel C, Losser MR. Is thenar tissue hemoglobin oxygen saturation in septic shock related to macrohemodynamic variables and outcome? Crit Care 2009; 13 (Suppl 5): S6.

70. Shapiro NI, Arnold R, Sherwin R, O'Connor J, Najarro G, Singh $S$, et al. The association of near-infrared spectroscopy-derived tissue oxygenation measurements with sepsis syndromes, organ dysfunction and mortality in emergency department patients with sepsis. Crit Care 2011; 15: R223.

71. Lima A, van Genderen ME, Klijn E, Bakker J, van Bommel J. Peripheral vasoconstriction influences thenar oxygen saturation as measured by near-infrared spectroscopy. Intensive Care Med 2012; 38: 606-11.

72. Colin G, Nardi O, Polito A, Aboab J, Maxime V, Clair B, et al. Masseter tissue oxygen saturation predicts normal central venous oxygen saturation during early goaldirected therapy and predicts mortality in patients with severe sepsis. Crit Care Med 2012; 40: 435-40.

73. De Backer D, Ospina-Tascon G, Salgado D, Favory R, Creteur J, Vincent JL. Monitoring the microcirculation in the critically ill patient: current methods and future approaches. Intensive Care Med 2010; 36: 1813-25.

74. Balestra GM, Bezemer R, Boerma EC, Yong ZY, Sjauw $\mathrm{KD}$, Engstrom $\mathrm{AE}$, et al. Improvement of sidestream dark field imaging with an image acquisition stabilizer. BMC Med Imaging 2010; 10: 15.

75. Sakr Y, Dubois MJ, De Backer D, Creteur J, Vincent JL. Persistent microcirculatory alterations are associated with organ failure and death in patients with septic shock. Crit Care Med 2004; 32: 1825-31.

76. Trzeciak S, Dellinge RP, Parrillo JE, Guglielmi M, Bajaj J, Abate NL, et al. Early microcirculatory perfusion derangements in patients with severe sepsis and septic shock: relationship to hemodynamics, oxygen transport, and survival. Ann Emerg Med 2007; 49: 88-98.

77. Ruiz C, Hernández G, Godoy C, Downey P, Andresen M, Bruhn A. Sublingual microcirculatory changes during highvolume hemofiltration in hyperdynamic septic shock patients. Critical Care 2010; 14: R170.

78. Dubin A, Pozo MO, Casabella CA, Murias G, Pálizas F, Moseinco MC, et al. Comparison of $6 \%$ hydroxyethyl starch 130/0.4 and saline solution for resuscitation of the microcirculation during the early goal-directed therapy of septic patients. J Crit Care 2010; 25: 659.e1-e8.

79. Sadaka F, Aggu-Sher R, Krause K, O’Brien J, Armbrecht ES, Taylor RW. The effect of red blood cell transfusion on tissue oxygenation and microcirculation in severe septic patients. Ann Intensive Care 2011; 1: 46.

80. Spronk PE, Ince C, Gardien MJ, Mathura KR, Oudemans-van Straaten HM, Zandstra DF. Nitroglycerin in septic shock after intravascular volume resuscitation. Lancet 2002; 360: 1395-6.

81. Boerma EC, Koopmans M, Konijn A, Guglielmi M, Bajaj J, Abate NL, et al. Effects of nitroglycerin on sublingual microcirculatory blood flow in patients with severe sepsis/septic shock after a strict resuscitation protocol: a double-blind randomized placebo controlled trial. Crit Care Med 2010; 38: 93-100.

82. Hernández G, Bruhn A, Castro R, Regueira T. The holistic view on perfusion monitoring in septic shock. Curr Opin Crit Care 2012; 18: 280-6.

83. Salazar N, Griñen H, Jirón M, Rojas L, Escobar L, Berasaín MA, Romero CM. Impacto del cuidado multidisciplinario en los desenlaces clínicos de los pacientes críticos. Rev Chil Med Intensiv 2012; 27: 15-22. 